\title{
Rankine Cycles, Modeling and Control
}

\author{
Ylva Teleman ${ }^{1}$ Pieter Dermont ${ }^{2}$ Hak Jun Kim ${ }^{3}$ Kil Sang Jang ${ }^{3}$ \\ ${ }^{1}$ Faculty of Engineering (LTH), Lund University, Sweden, ylva.teleman@gmail . com \\ ${ }^{2}$ Modelon AB, Sweden, pieter. dermontemodelon.com \\ ${ }^{3}$ Hanon Systems, South Korea, $\{$ hkim18, kjang1 $\}$ @hanonsystems . com
}

\begin{abstract}
As the need for increased energy efficiency grows, the use of new energy sources is a topic of investigation for research and industrial applications. The ability to use low temperature heat sources via a Rankine or organic Rankine cycle is one of the options. In this paper such a cycle is modelled and simulated using a Modelica based thermal management library suite as well as the simulation tool Dymola. Experimental test bench data provided by Hanon Systems allowed calibration and verification of the simulation results. Simulation results shows good agreement with experimental data. Additional dynamic simulations are performed to illustrate potential applications of the model for system optimization and control development.

Keywords: Rankine cycles, organic Rankine cycles, modeling, dynamic simulations, Modelica, Dymola, thermofluid
\end{abstract}

\section{Introduction}

\subsection{Rankine Cycle}

A Rankine cycle is a thermodynamic cycle which utilizes heat to create mechanical power, harvested by an expander that can drive a generator. The medium of the Rankine cycle, usually water, is pressurized in a pump, evaporated in a heat exchanger, subsequently passed through an expander and finally condensed in condenser. The process and essential components in the cycle can be seen in Figure 1. By choosing a medium with appropriate properties, different heat source temperatures can be used.

\subsection{Purpose}

This paper is a result of an academic collaboration with the Faculty of Engineering (LTH) of Lund University. The purpose of the project was to model and simulate an industrial use-case: a Rankine cycle that utilizes waste heat from a combustion engine vehicle to generate mechanical power. The project scope was defined by Mod-

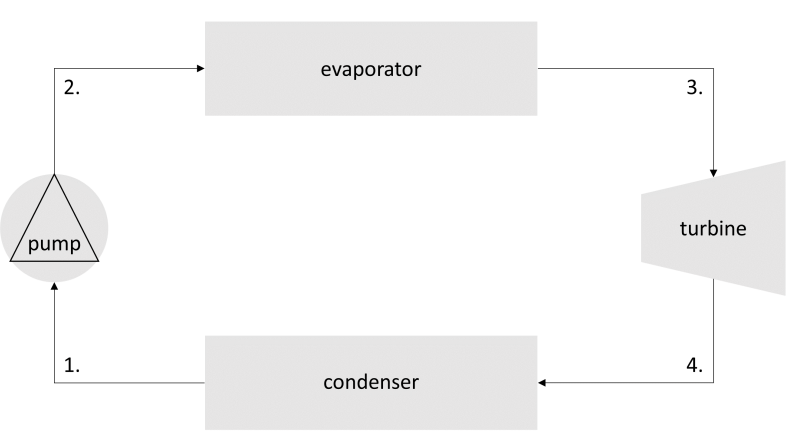

Figure 1. Essential components in a Rankine cycle.

elon, a company specialized in simulation and optimization using open standard technology. The aim of the project was to model or use existing models of components, parameterize the components, build the complete cycle, implement appropriate control and gain knowledge of the cycle's behavior.

\subsection{Thermofluid Modeling}

The models were implemented in Modelica, an open standard modeling language (ModelicaAssociation, 2015), using a 1-D thermofluid approach. The principles of thermofluid modeling using Modelica are laid out in (Tummescheit, 2002; Eborn, 2001).

The different components were either selected from preexisting model libraries or modeled. A specialized thermal management suite was used, based on three compatible model libraries: Vapor Cycle library (Modelon, 2015c), Heat Exchanger Library (Modelon, 2015a; Batteh et al., 2014) and Liquid Cooling Library (Modelon, 2015b; Batteh et al., 2014).

The modeling was performed in the simulation environment Dymola 2016 FD01(DassaultSystèmes, 2015).

\subsection{Method}

After the physical components were either selected or modeled and calibrated, they are connected to create the complete cycle. Simulations with different control 
strategies and conditions were tested. In order to verify results, data received from Hanon Systems was used. Hanon Systems is formerly known as Halla Visteon Climate Control and specializes in thermal management solutions for automotive applications. The components and cycle were parameterized according to 11 experimental data sets.

\section{System Presentation}

\subsection{Refrigerant}

A refrigerant called $\mathrm{R} 134 \mathrm{a}$, also known as 1,1,1,2tetrafluoroethane, is the working fluid of the cycle. While utilizing low grade waste heat is most commonly done using an organic refrigerant, the waste heat temperature is sufficiently high in Hanon System's test setup to use a conventional refrigerant.

Results are repeatedly represented in the specific enthalpy - pressure diagram of the working fluid. The diagram for R134a can be seen in Figure 2. Within the thumb-like shape, the refrigerant is a two-phase fluid, a mixture of both liquid and gas. To the left and right of the dome, only liquid respectively vapor exists. The isotherms transverse the two-phase dome horizontally.

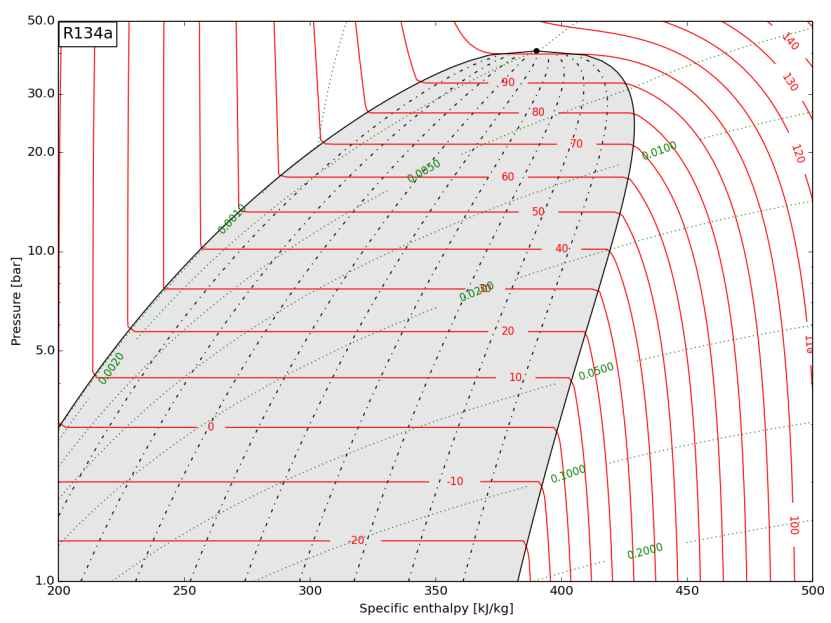

Figure 2. Pressure/enthalpy diagram for R134a.

\subsection{Components}

All but one component used for the Rankine cycle were readily contained in one of Modelon's thermal management libraries suite. Hanon Systems' test bench utilizes a positive displacement machine, more specifically a diaphragm pump that was implemented as a custom component.

A diaphragm pump operates conceptually similar to a human diaphragm does - a chamber and a membrane that moves outward sucking working fluid into the space or move inwards in order to push out the working fluid. The pump has a $4.46 \mathrm{~kW}$ power output at $1750 \mathrm{rpm}$. Its losses can be described through three distinct efficiencies; volumetric, isentropic and mechanical. The volumetric efficiency describes ratio of the volume in the pump that is displaced to the geometric volume. The isentropic efficiency describes how much energy is lost during the process of pressuring, and the mechanical efficiency describes losses in shaft and other components of the pump. The efficiencies of the pump were estimated with the experimental data, partly relying on assumptions since data for the fluid at the outlet of the pump was missing.

The expander used in Hanon Systems' cycle is a positive displacement machine: a scroll turbine. It has two intertwined spirals, one stator and one rotor. The hot high pressured gas enters in the middle, pushing the rotor around as it makes its journey between the spirals until it at last exits the positive displacement machine. Similar to the pump, the expander is subject to losses and they are described using equivalent efficiencies; volumetric, isentropic and mechanical. Again, these efficiencies were calculated from the data provided by Hanon Systems.

The cycle includes a plate heat exchanger which acts as evaporator, using glycol on the secondary side. Not all geometry parameter data was available, and the missing parameters were estimated or set to typical values. A detailed geometry-based plate heat exchanger model exists in the model libraries, with a higher number of states due to its flow configuration. A less complex counterflow heat exchanger model with equivalent parameterization was used assuring fast solver convergence but less accurate behavior representation. In Table 1 some geometry data provided by Hanon Systems for the plate heat exchanger can be seen.

\begin{tabular}{c|c} 
Item & Value \\
\hline Type & MCV Plate \\
\# plates & 36 rows \\
Fin & N.A. \\
Path & $6-12-18$ \\
Effective size mm & $93.1 * 170.5 * 70.4$
\end{tabular}

Table 1. Plate heat exchanger data

The condenser of the cycle is a flat tube heat exchanger using ambient air to cool down the working fluid. A detailed geometry-based heat exchanger model was used, parameterized with both available geometry data and typical geometry data. In Table 2 some geometry data provided by Hanon Systems for the flat tube heat exchanger can be seen.

A tank is integrated in the cycle between condenser and pump. The purpose of the tank is twofold: (1) ensure only liquid entered the pump as gas would damage it and (2) balance the amount of working fluid in the cycle. A static head of $1 \mathrm{~m}$ was introduced between condenser and pump to confirm that the dynamic pressure at the inlet of the pump was sufficiently high to avoid the creation of bubbles in the fluid. The tank has a $8 \mathrm{~L}$ volume. 


\begin{tabular}{c|c} 
Item & Value \\
\hline Type & SC $20 \mathrm{t}$ \\
\# tubes & 54 rows \\
Fin & 80 fpdm \\
Path & $44-10$ \\
Effective size mm & $570 * 383$
\end{tabular}

Table 2. Flat tube heat exchanger data

\subsection{Control}

Based on the cycle configuration different control strategies are relevant. The following strategies were considered:

- Adequate super heating to let only gas into the turbine

- Adequate sub cooling or other measure to avoid gas entering the pump

- Preferred power or torque from the turbine

- Optimal evaporation temperature

- Low condenser pressure

- Appropriate amount of refrigerant in the cycle

Except for choosing appropriate components that will match the requirements, it is possible to change the following variables (Quoilin et al., 2011):

- The speed of the turbine

- The speed of the pump

- Add tanks after condenser/evaporator

- Drain or charge the cycle with WF

- Change the temperature of the heat source for the evaporator

- Change the temperature of the cooling air in the condenser

The temperature of the heat source in the evaporator as well as the cooling air in the condenser are typically not entities that can be controlled in automotive applications as the heat source typically is the heat from the engine and the temperature of the air is dependent on ambient conditions. It is however of interest to gain knowledge about the behavior of the cycle under different conditions.

The speed of the pump has a direct consequence on the amount of superheat of the working fluid leaving the evaporator. Decreasing the speed of the pump, the amount of superheating is increased. Effective superheat control is important to ensure no two-phase fluid enters and potentially damages the expander. The speed of the expander was varied in order to achieve desired power or torque as they are correlated according to Equation 1.

$$
\operatorname{Torque}(\mathrm{Nm})=\frac{\operatorname{Power}(W)}{\operatorname{Speed}(\mathrm{rad} / \mathrm{s})}
$$

For both superheat and torque control PI-controllers were used.

In this project the heat source and temperature of cooling air were kept constant. The overall efficiency is calculated according to Equation 2.

$$
\eta_{\text {overall }}=\frac{W_{\text {turbine }}-W_{\text {pump }}}{Q_{\text {evaporator }}}
$$

\subsection{Initialization}

Initialization is important to ensure a fast solver convergence. In this cycle, the most complex components are the heat exchangers. Since the heat exchangers contain the majority of continuous time states, their initialization is key. Additionally, it is necessary to set the PIcontroller parameters correctly in order to achieve robust control.

The amount of working fluid in the cycle can be changed either by:

- Changing the initialization of the cycle, i.e. the continuous times states of the thermofluid model, which results in a refrigerant mass.

- Directly setting the tank level.

- Adding a charge component that can either charge or drain the cycle to a desired amount of working fluid during simulation.

\subsection{Data}

Hanon Systems provided 11 data sets that varied in the amount of working fluid and super heating. For every data set the speed, power and torque were measured for pump and turbine, and inlet and outlet pressure and temperature were measured for all the components (with exception of outlet of pump).

On the test bench test conditions such as speed of the pump, ambient temperature, air velocity, mass flow and boiler coolant temperature were controlled. The speed of the expander was controlled such that the torque remained constant at $9 \mathrm{Nm}$, and a tank was placed between the condenser and the pump. Controls for dynamic operation of the cycle weren't implemented at the time of the measurements. However, the maximum power of the expander was determined by changing expander brake torque for different pump speeds and air temperatures in the condenser. 


\subsection{Test Scenarios}

The following control strategies or conditions were tested for the cycle:

- The different data sets were simulated.

- The cycle was tested with different torques on the turbine to match the maximum power point tracking diagram Hanon Systems had implemented. Hanon Systems concluded from experimental data that maximum power is achieved at $9 \mathrm{Nm}$ brake torque.

- Superheat control was tested.

- The cycle was tested with varying amounts of refrigerant.

\section{Creating the Model}

\subsection{Expander}

The isentropic efficiency for the expander was calculated using the data. For all the data sets it varied between $43-$ $55 \%$ depending on pressure ratio and speed. No correlation could however be demonstrated, likely due to measurement errors and thus a mean value was used. When calculating the mechanical efficiency, only 3 of the 11 data sets give values under $100 \%$. For 9 of the data sets the mass flow was too low, and it was assumed that an error in either the data or the measuring of data had occurred. Consequently a mean value of the mechanical efficiency from the three good data sets was used and resulted in a base value of $87 \%$.

\subsection{Pump}

Unlike the expander, the pump's efficiencies were mapped in a grid depending on speed and pressure ratio. Since it was known that the mass flow was inconsistent for 9 of the data sets, only the three data sets with consistent data were used to calculate the efficiencies. The mechanical efficiency was set to $73 \%$, and the isentropic ranged between $58 \%$ and $70 \%$ depending on speed and pressure ratio over the pump.

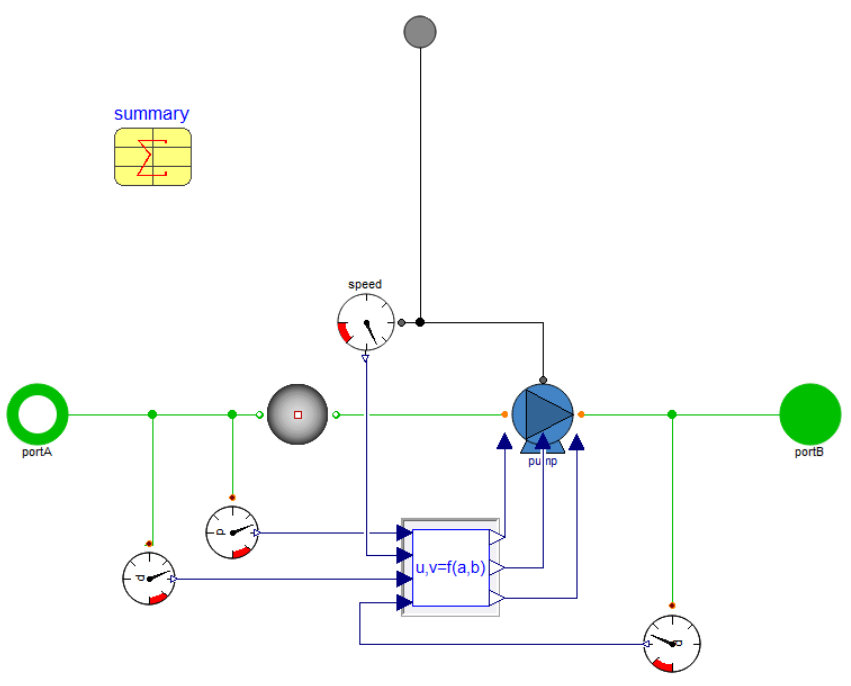

Figure 3. The model of the pump as seen in Dymola.

The top level class of the pump in the Dymola diagram layer is illustrated in Figure 3. The model is based on a generic pump model from the Liquid Cooling Library, in which a function prescribes the pump's behavior. Through an interface, this function can be exchanged. The speed of the shaft, density at the inlet and pressure at the inlet, and at the outlet are inputs to the interface. Outputs of the interface are isentropic and mechanical efficiencies, as well as mass flow. To create a diaphragm pump, appropriate behavior-prescribing tables dependent on speed and pressure ratio were inserted in the interface, and the efficiencies are then extrapolated from the tables. The interface additionally calculates the mass flow based on the density at the inlet, speed, maximum displacement volume, and volumetric efficiency.

\subsection{Heat Exchangers}

In order to calibrate the heat exchangers, virtual test benches were set up. In these virtual test benches, the heat exchanger was connected to mass flow and pressure source components (Figure 4). After setting geometry parameters, applying correct boundary conditions, and selecting appropriate heat transfer and pressure drop correlations, the calibration factors were used to calibrate the heat exchanger model. The calibration factors tune the pressure drop as well as the heat transfer coefficients.

\subsection{Complete Cycle}

The complete assembled cycle in the Dymola diagram layer is illustrated in Figure 5. The cycle contains a simple evaporator, a tank between the condenser and pump, and includes all necessary control for dynamic simulation. 

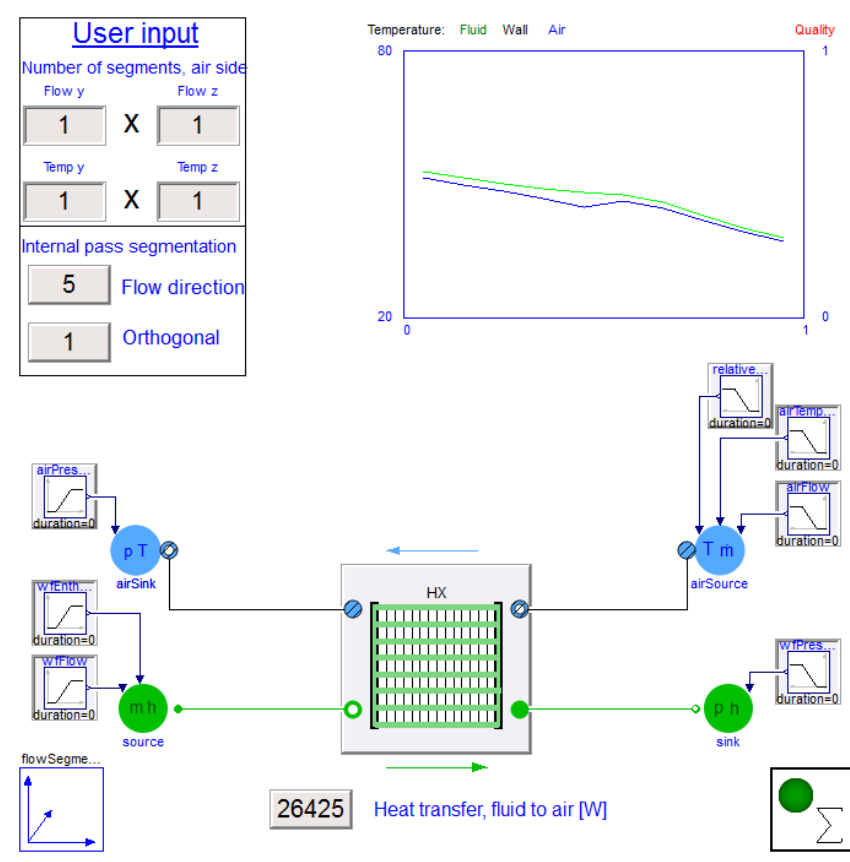

Figure 4. Virtual testbench for the condenser. It was based on a template with a default heat exchanger and boundary condition parameterization (HeatExchanger.HeatExchangers.FlatTube.Experiments. TestBenches.AirTwoPhaseHomogeneous).

\section{Results}

The result section is composed of two parts. The first part validates the model by comparison with the experimental data and demonstrates that the model can mimic its physical counterpart under static conditions. A comparison of a relevant cycle metric is performed: a power tracking diagram is plotted. Secondly, the dynamic capabilities of the model are shown through a set of simulation experiments. Simulations took between 2 and 10 min for a cycle with the simple evaporator, and $0.5-1.5 \mathrm{~h}$ with the complex one.

\subsection{Simulation of Different Data Sets}

All 11 data sets, each with different boundary conditions, matched the simulation results well. The sets with coherent data resulted in the best match as expected. In Figure 6 two specific enthalpy - pressure diagrams with thermodynamic cycle are depicted; on the left is the cycle constructed with experimental data of data set 10 and on the right the cycle as obtained from the simulation using data set 10 boundary conditions.

In Table 3 and 4 simulation results as well as experimental data are compared. The simulation of data set 10 is more accurate than the one of set 9; data set 10 had coherent data and set 9 did not.

\begin{tabular}{c|c|c} 
Data set & 9 & Simulation \\
\hline Power W & 834 & 836 \\
Overall efficiency \% & 2.2 & 1 \\
Mass flow kg/s & 0.12 & 0.12 \\
SH ${ }^{\circ} \mathrm{C}$ & 2.5 & 2.5 \\
Speed of turbine rpm & 885 & 886 \\
Speed of pump rpm & 350 & 343 \\
Torque of turbine Nm & 9 & 9 \\
Turbine pressure ratio & 1.87 & 2.03 \\
Pressure ratio error \% & - & 8.6 \\
Isentropic efficiency turbine & - & 61
\end{tabular}

Table 3. Simulation results for data set 9 .

\begin{tabular}{c|c|c} 
Data set & 10 & Simulation \\
\hline Power W & 820 & 827 \\
Overall efficiency \% & 1.7 & 1.8 \\
Mass flow kg/s & 0.138 & 0.138 \\
SH ${ }^{\circ} \mathrm{C}$ & 0.5 & 0.5 \\
Speed of turbine rpm & 870 & 879 \\
Speed of pump rpm & 400 & 406 \\
Torque of turbine Nm & 9 & 9 \\
Turbine pressure ratio & 1.89 & 1.84 \\
Pressure ratio error \% & - & 2.6 \\
Isentropic efficiency turbine & - & 60
\end{tabular}

Table 4. Simulation results for data set 10.

\subsection{Maximum Power Tracking Diagram}

The graph in Figure 7 illustrates the behavior of the power harvested by the expander as a function of the torque applied to the expander. Experimental and simulation data match well. The largest difference is approximately $3 \%$.

\subsection{Superheat Control}

Dynamic superheat control setpoint control response is plotted in Figure 8. The super heating was measured by the sensor component in Figure 5. The output was then sent to the PI-controller which compares the measured value with the desired value and subsequently adjusted the pump speed.

\subsection{Refrigerant charge}

The refrigerant source components allows charging or draining the cycle during the simulation. It was demonstrated that overcharging the cycle results in any increase of pressure in all components with a ultimately a loss of pressure difference between evaporator and condenser. Similar observations could be made for a starved cycle where the pressure in components decreases as illustrated in Figure 9. Expander power loss was significant, ranging from approximately $850 \mathrm{~W}$ with sufficient refrigerant charge, to $137 \mathrm{~W}$ for a starved cycle. 


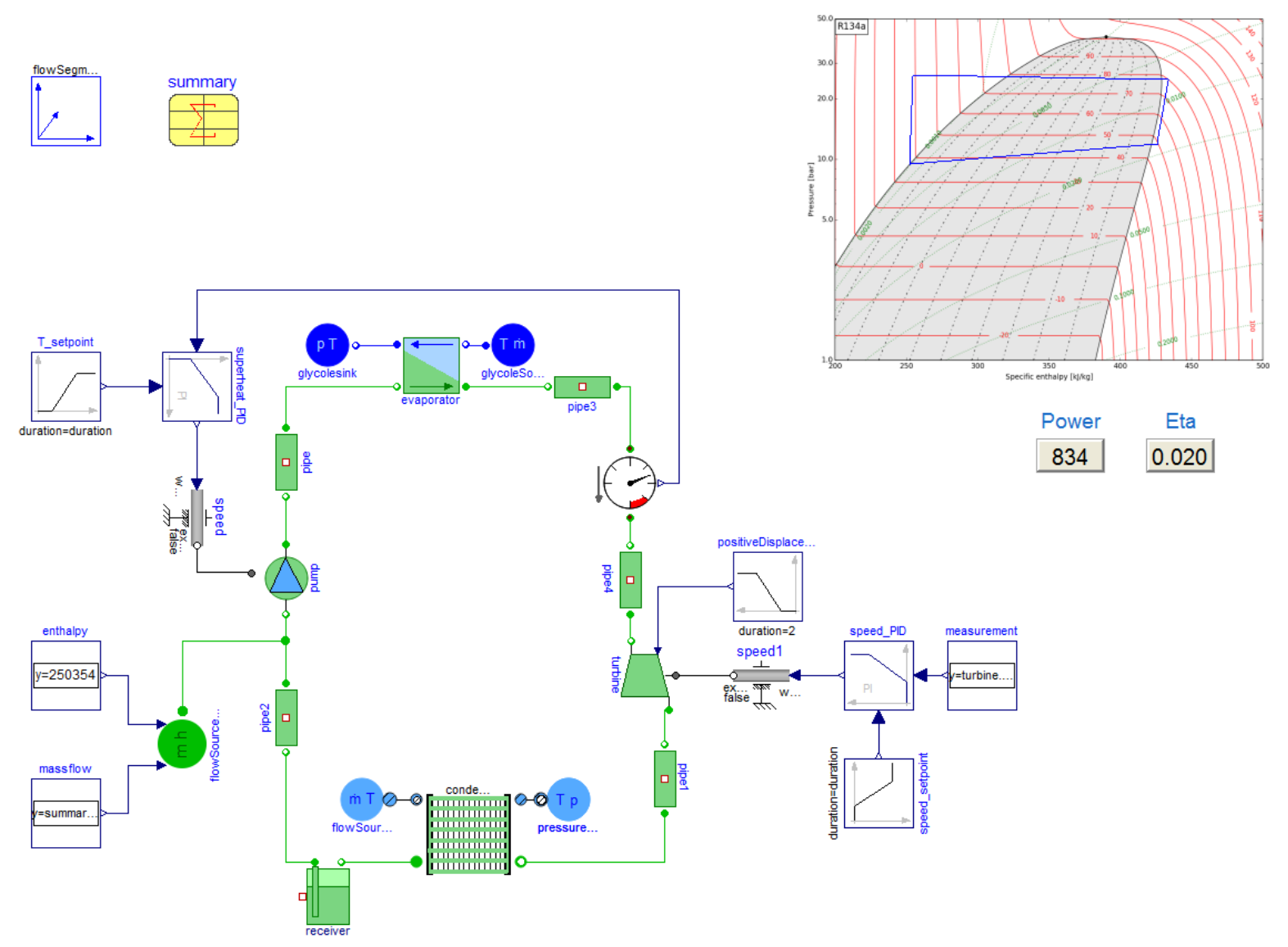

Figure 5. The whole Rankine cycle as seen in Dymola.
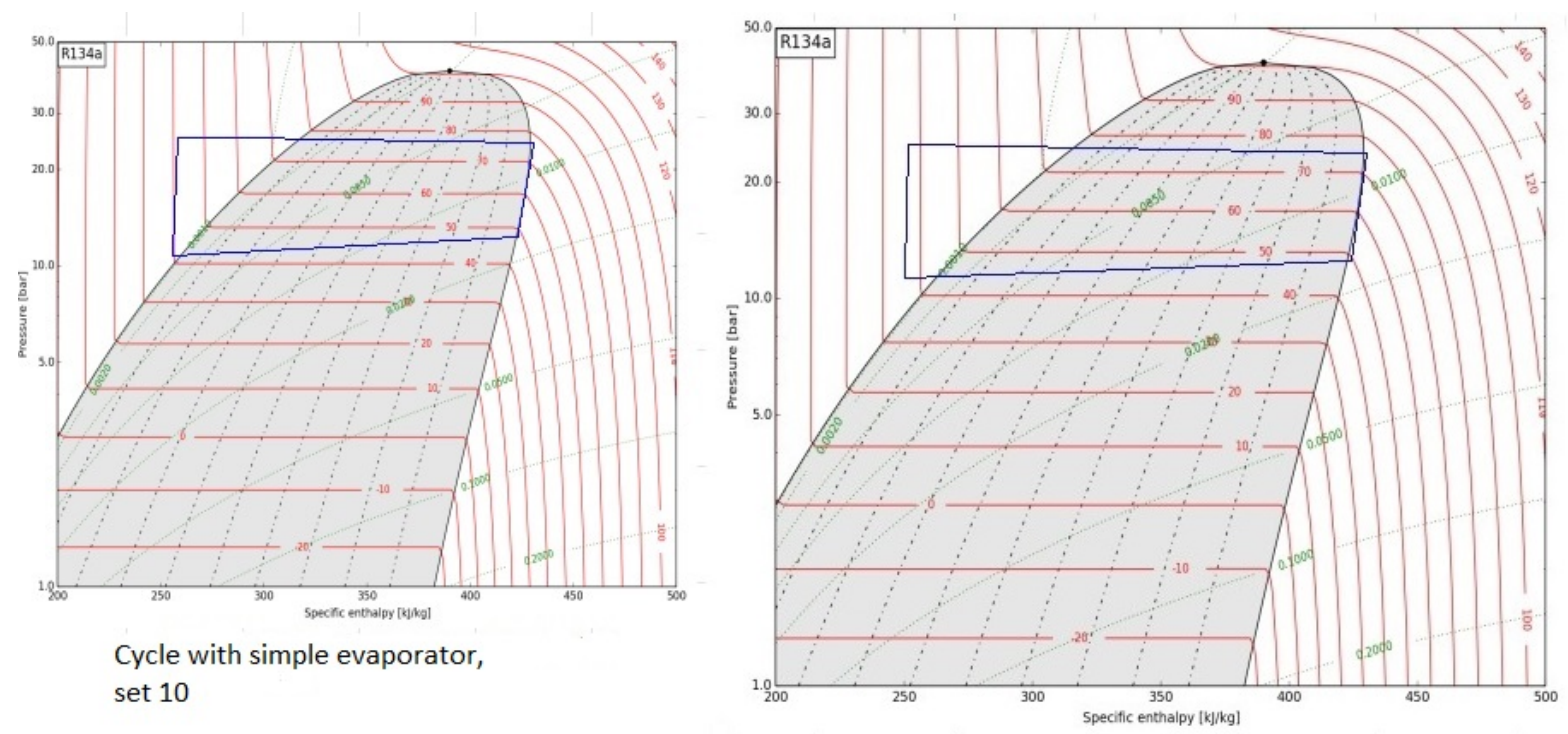

Figure 6. Simulation result for data set 10, on a pressure/enthalpy diagram to the left, data to the right.

\section{Discussion and Conclusion}

A Rankine cycle was modeled and parameterized according to data from Hanon Systems, and control strategies were implemented to gain insight into the behavior of the cycle and for controls virtual prototyping. Some variables had to be estimated since data was missing or inaccurate.

Steady state simulation results matched experimental data well. Additional geometrical information on individual components as well as the piping between components would allow to create a more trustworthy model, 


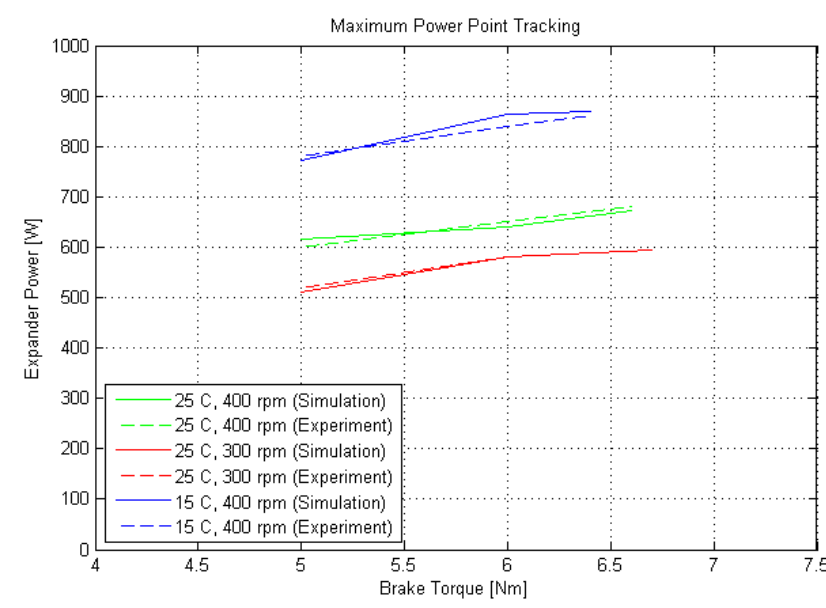

Figure 7. Maximum power point diagram, comparison of experimental and simulation data
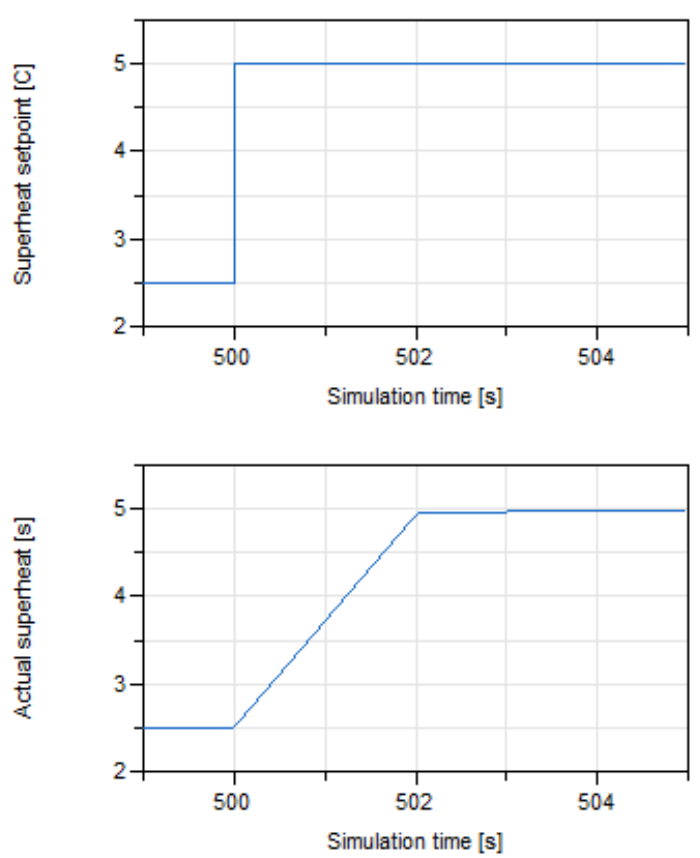

Figure 8. Superheat control. Setpoint and system response.

with an accurate total volume and all pressure drops included. The power point tracking diagram measured out by Hanon Systems is created with the model with only small deviations of the expander power output.

The control strategies that were implemented and the initialization worked effectively. At the point of completion of the model, no control was specified for the system test bench. Dynamic simulations were carried out based on typical control mechanisms for Rankine cycles.

The cycle created in this project was a generic and simple one intended for automotive applications. In order to gain more knowledge about industrial Rankine cycles different setups should be modeled and simulated. The overall efficiency of the cycle varied between approximately 1.7 and $2.2 \%$. Hanon Systems' primary in-

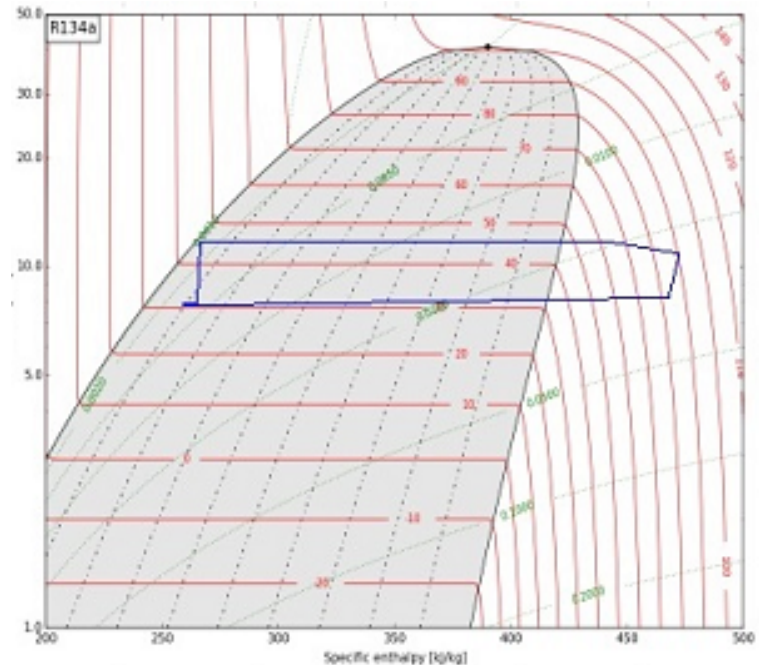

Figure 9. Thermodynamic cycle after drain to a specific charge of $300 \mathrm{~kg} / \mathrm{m}^{3}$ - turbine power output is at $137 \mathrm{~W}$.

tention with the test bench was to focused on the development and testing of an expander. Higher efficiencies can be achieved with state-of-the-art Rankine cycles.

\section{References}

John Batteh, Jesse Gohl, and Sureshkumar Chandrasekar. Integrated vehicle thermal management in modelica: Overview and applications. In Proceedings of the 10th International Modelica Conference, 2014.

DassaultSystèmes. Dymola 2016 fd01, 2015. URL http://www.3ds.com/products-services/ catia/products/dymola.

Jonas Eborn. On model libraries for thermo-hydraulic applications. PhD thesis, Lund University, 2001.

ModelicaAssociation, 2015. URL www . modelica.org.

Modelon.
2015a. products/modelica-libraries/ heat-exchanger-library/. Modelon. $\quad$ Liquid cooling library,
2015b. URL products/modelica-libraries/ liquid-cooling-library/.

Modelon. Vapor cycle library, 2015c. URL www . modelon. $\mathrm{com} / \mathrm{products/modelica-libraries/}$ vapor-cycle-library/.

Sylvain Quoilin, Richard Aumann, Andreas Grill, Andreas Schuster, Vincent Lemort, and Hartmut Spliethoff. Dynamic modeling and optimal control strategy of waste heat recovery organic rankine cycles. Applied Energy, 88(6): 2183-2190, 2011. 
Hubertus Tummescheit. Design and implementation of objectoriented model libraries using modelica. PhD thesis, Lund University, 2002. 